\title{
Diseño y propiedades psicométricas de un instrumento para evaluar habilidades de comprensión lectora en estudiantes de tercer año básico*
}

\author{
Development and psychometric properties of a reading \\ comprehension skill test for third grade students \\ Construção e propriedades psicométricas de um instrumento \\ para avaliação compreensão de leitura de alunos do terceiro ano \\ Alfaro Urrutia, Jorge Eduardo ${ }^{a}$, Santibáñez Riquelme, Juan Domingo ${ }^{b}$ \\ aUniversidad de la Frontera, Temuco, Chile. \\ Teléfono: +56 9 77571688. Correo electrónico: jorgeeduardoau@gmail.com \\ bUniversidad de Concepción, Concepción, Chile. \\ Teléfono: +5641240 5196. Correo electrónico: jsantiba@udec.cl
}

\begin{abstract}
RESUMEN
El objetivo de la investigación es diseñar y analizar las características psicométricas de un instrumento para evaluar habilidades de comprensión lectora en estudiantes de tercer año básico de establecimientos municipales de la ciudad de Puerto Montt. El instrumento se diseñó utilizando un análisis de redes semánticas naturales y revisión bibliográfica. La validez de contenido se estimó a partir de juicio de expertos. La muestra estuvo conformada por 1.000 estudiantes. El instrumento final cuenta con 21 ítems que el análisis factorial exploratorio agrupó en seis factores: extracción de información, habilidades fonológicas, estrategias durante la lectura, estrategias después de la lectura, interpretación de textos no literarios y secuenciación. La confiabilidad del instrumento resultó adecuada con un Alfa de Cronbach de .778 y se encontraron correlaciones significativas entre el puntaje del instrumento y las calificaciones de la asignatura de lenguaje, test de autoestima escolar y necesidades educativas especiales de los estudiantes.
\end{abstract}

Palabras clave: comprensión lectora, validación, confiabilidad.

\begin{abstract}
This study aims to develop and analyze the psychometric properties of a reading comprehension skills test for third grade students in Puerto Montt, Chile. The test foundations come from the analysis of natural semantic networks and a bibliographical revision and an expert panel was in charge of validating the test's content. 1000 third grade students were evaluated independently. The final version of the instrument contained 21 items which, through exploratory factor analysis, were grouped in six factors: information extraction, phonological skills, during reading strategies, after reading strategies, non-literary texts interpretation, and sequencing. Internal reliability of the test was appropriate (alpha=.778), and significant correlation between the score of the instrument, the score obtained in the language subject, the academic self-esteem test, and the educational special needs of the students was found.
\end{abstract}

Key words: reading comprehension, validation, reliability.

\footnotetext{
* $\quad$ Este estudio surge a partir de la Tesis de Magíster "Diseño y propiedades psicométricas de un instrumento para evaluar habilidades de comprensión lectora en estudiantes de tercer año básico” de la Universidad de La Frontera.
} 


\section{RESUMO}

Propõem-se a construção e análise das características psicométricas de um instrumento para avaliar habilidades de compreensão da leitura de alunos do terceiro ano básico em estabelecimentos municipais da cidade de Puerto Montt, Chile. Utilizaram-se a técnica das redes semânticas naturais e revisão bibliográfica. A validade de conteúdo se deu por meio da opinião de especialistas. O instrumento foi aplicado a uma amostra de 1000 alunos do terceiro ano. $\mathrm{O}$ instrumento final conta com vinte e um itens agrupados pela análise fatorial exploratória em seis fatores: extração de informações, habilidades fonológicas, estratégias durante a leitura, estratégias depois da leitura, interpretação de textos não literários e sequenciação. A consistência do instrumento segundo o Coeficiente Alfa de Cronbach de 0,778 é adequada e foram encontradas correlações significativas entre a medição do instrumento e as qualificações da disciplina de linguagem, teste de autoestima escolar e necessidades educativas especiais dos estudantes.

Palavras chave: compreensão de leitura. validação. confiabilidade.

\section{INTRODUCCIÓN}

El problema de la comprensión lectora se encuentra ampliamente estudiado. Debido a que su implicancia en el aprendizaje, en el éxito académico y en el acceso al conocimiento, la información y la cultura resulta fundamental, se ha incrementado de manera importante la preocupación por evaluarla y conceptualizarla en los últimos años (OCDE, 2003; MINEDUC, 2009; Rosas, Medina, Meneses, Cuchacovich y Escobar, 2011; IEA, 2012; Allende, Condemarín y Milicic, 2012; Marchant, Recart, Cuadrado y Sanhueza, 2012; Bizama, Arancibia y Sáez, 2013; Soriano-Ferrer, Sánchez-López, Soriano-Ayala y Nievas-Cazorla, 2013; Canet-Juric, Burin, Andrés y Urquijo, 2013; Pascual, Goikoetxea, Corral, Ferrero y Pereda, 2014; Ripoll y Aguado, 2014).

De forma clásica se ha entendido la comprensión lectora como el conocimiento de los significados de las palabras, el significado de las oraciones y la interpretación de las ideas e intenciones del texto (García, 1993). De una forma más precisa, se establece la comprensión lectora como una "habilidad para extraer significados del texto" (Rathyon, 2004:156). Así, la comprensión lectora se sitúa como una habilidad que, además de implicar el conocimiento de ciertos elementos aislados, implica la comprensión de una idea global. Barreyro y Molinari (2005) refuerzan esta idea definiéndola como la construcción en la memoria episódica de una representación mental del texto. Finalmente, Gómez, Defior y Serrano (2011) señalan que la comprensión lectora es el objetivo final de la lectura a partir de procesos que van desde la lectura de palabras, oraciones, hasta comprender su contenido global.

La Organización de las Naciones Unidas para la Educación, Ciencia y la Cultura, UNESCO (2008), define y explica la lectura como un proceso en el que se ponen en desempeño un conjunto de habilidades que permiten no solo relacionar una información literal del texto, sino a una diversidad de datos. Identifica así procesos generales (reconocer información literal, inferir información, relacionar datos separados, discriminar datos, sintetizar información, generalizar, reconocer sentidos figurados y analizar unidades mínimas de significado), procesos relativos a textos específicos (determinar intenciones de un personaje, identificar subtemas, reconocer explicación científica) y procesos metalingüísticos (denominar partes de un texto, reconocer tipos de texto, identificar macroestructuras).

El informe del Programa Internacional para la Evaluación de Estudiantes (PISA) (OCDE, 2013) define la comprensión lectora como entender, usar, reflexionar e implicarse 
con los textos escritos, en términos de lograr objetivos personales, desarrollar el propio conocimiento y participar en la sociedad. Identifica seis niveles desde las habilidades más básicas a las más complejas, entre ellas se encuentran en orden creciente de complejidad la identificación de información explícita, la localización de información, relacionar conocimientos previos, clasificar información, formular hipótesis, realizar inferencias, reflexionar acerca de la lectura.

Gutiérrez-Braojos y Salmerón (2012) identifican tres momentos en que se desarrollan estrategias de comprensión lectora, antes, durante y después de la lectura. Entre las habilidades específicas señalan el identificar la finalidad de la lectura, la activación de conocimientos previos, la identificación de palabras desconocidas, relectura, representación visual, formular inferencias, detectar información relevante, construcción global y finalidad comunicativa.

La comprensión lectora, así, consiste en un conjunto de habilidades que van desde aspectos como la decodificación y significación de las palabras hasta la extracción de ideas, relación y reflexión concerniente a lo leído con una finalidad personal. Se amplía de esta forma el concepto que tradicionalmente se tiene de este fenómeno.

El Ministerio de Educación chileno (MINEDUC, 2013), en el marco de la Ley General de Educación del año 2010, actualiza sus programas de estudio en el área de Lenguaje y Comunicación organizándolos según habilidades, conocimientos y actitudes. Define las habilidades como una capacidad para realizar una tarea y para solucionar un problema de forma precisa y adaptable. Entre los objetivos de aprendizaje que establece para tercer año básico se identifican varias habilidades como la decodificación, entonación, velocidad, relacionar conocimientos previos, relectura, visualización, formular y responder preguntas, subrayar, extraer información explícita e implícita, descripción, expresión de opiniones, interpretación de lenguaje figurado, localizar información, usar imágenes, expresar opiniones y determinar significados desconocidos.

La fluidez lectora se define como una habilidad para leer palabras, pseudopalabras y textos con precisión de forma expresiva y a ritmo apropiado (Gómez et al., 2011). Se ha establecido que problemas en la decodificación conllevan a dificultades posteriores en la comprensión lectora y en el aprendizaje en general (Paredes, 2006; Marchant, Lucchini y Cuadrado, 2007; Muñoz y Schelstraete, 2008).

Ahmadi (2012) identifica habilidades de comprensión lectora que facilitan al estudiante el comprender las ideas, el scanning que identifica como una técnica en donde el estudiante busca información específica en el texto, la adivinación de significados en la que el estudiante utiliza el contexto, la estructura de la palabra o sus conocimientos previos acerca del tema para determinar aquellos significados que no conoce, el automonitoreo que le permite al estudiante formular preguntas e interrogantes respecto de lo que está leyendo y finalmente las inferencias que pueden ser de diverso tipo, entre ellas el establecer relaciones gramaticales implícitas, intenciones de los personajes, llenar vacíos en el texto o identificar la intención del texto (Cassany, Luna y Sanz, 2000; Barreyro y Molinari, 2005; Escudero, 2010).

La falta de desarrollo en estas habilidades podría conllevar a presentar dificultades generales o específicas en el aprendizaje de la lectura que impactan en el aprendizaje y en la participación de los estudiantes en su vida escolar y social (Mora y Aguilera, 2000; Lyon, Shaywitz \& Shaywitz, 2003; Sánchez, 2008; Silvia e Aparecida, 2011; Goicoetxea, 2012). 
La conceptualización de la lectura solo como la decodificación sin considerar el desarrollo y práctica de las habilidades mencionadas anteriormente, deriva por tanto en bajos resultados en pruebas nacionales e internacionales en el área. En el 2012, el Centro Regional para el Fomento del Libro en América Latina y el Caribe (CERLAC, 2012) realizó un estudio en el que $20 \%$ de los chilenos no lee libros y el $32 \%$ no lee por desinterés. La Organización de las Naciones Unidas para la Educación, la Ciencia y la Cultura (UNESCO, 2008) realizó un estudio en Latinoamérica, donde Chile se encuentra una desviación estándar por sobre el promedio en comprensión lectora, ubicándose por sobre países como Brasil, El Salvador, Ecuador y Perú.

Sin embargo al comparar Chile con el resto del mundo los resultados son menos alentadores. La prueba PISA (MINEDUC, 2009) reporta que de 65 países participantes en la evaluación de lectura, Chile se ubica en el lugar número 44, con un promedio de 449 puntos, siendo el puntaje promedio de los participantes 493. El Sistema de Medición de la Calidad de la Educación SIMCE, consistente en una prueba (MINEDUC, 2012), reporta que el promedio en lectura es de 267 manteniéndose similar a la medición del año anterior. La región de Los Lagos, en donde se enmarca esta investigación, promedió en sus establecimientos municipales 255 puntos, mientras que el de los particulares subvencionados y particulares pagados promedió en 271 y 299.

Debido a la importancia que ha cobrado la comprensión lectora en los últimos años, la complejidad que ha representado su conceptualización y la de sus habilidades, además de los resultados reportados a nivel local, es que se considera que este estudio puede ser un aporte al campo científico en tanto pretende diseñar y analizar las características psicométricas de un instrumento que permita evaluar las habilidades de comprensión lectora de estudiantes de tercer año básico.

\section{MÉTODO}

Se trabajó desde un paradigma mixto utilizando un diseño no experimental exploratorio transversal.

\subsection{DISEÑO DEL INSTRUMENTO}

La primera fase del estudio se desarrolló sobre la base de una metodología cualitativa. Se utilizó el Análisis de Redes Semánticas Naturales (Vera-Noriega, 2005; Hinojosa, 2008) el que consiste en realizar un estudio de los significados que tienen ciertas palabras, conceptos o expresiones para un grupo social determinado. Se aplicó un cuestionario a 58 sujetos (9 hombres y 49 mujeres), entre ellos se encontraban educadores diferenciales, profesores de educación básica, asistentes sociales, psicólogos y psicopedagogos con un tiempo de ejercicio promedio de la profesión. Se consultó respecto del concepto de habilidades de comprensión lectora en contexto de tercer año básico, con la finalidad de conocer cuáles eran las habilidades que ellos estimaban necesarias para lograr un buen nivel de comprensión.

Asimismo, se realizó una revisión bibliográfica basada en los Objetivos de Aprendizajes del Currículum Chileno actualizado para Lenguaje y Comunicación (MINEDUC, 2013) 
y habilidades de comprensión lectora. A partir de ambos procesos (Análisis de Redes Semánticas y Revisión Bibliográfica) se realizó la construcción de una batería de textos y un conjunto de ítems para evaluar 17 habilidades de comprensión lectora.

El primer diseño del instrumento fue sometido a juicio de expertos utilizando el Método de Agregados Individuales (Corral, 2009), en el que participaron cuatro profesionales vinculados al área de educación de la comuna de Puerto Montt, entre ellos, una educadora diferencial mención en trastornos del aprendizaje, una asistente social y profesora básica, un profesor de educación básica con especialidad en Lenguaje y Comunicación y una psicóloga educacional. Cada profesional recibió dos pautas, la primera para evaluar los ítems y su forma de aplicación, y la segunda para evaluar la calidad de los textos de la batería a aplicar. Se establecieron porcentajes de aprobación por cada criterio.

\subsection{PROPIEDADES PSICOMÉTRICAS DEL INSTRUMENTO}

La segunda fase del estudio se desarrolló a base de una metodología cuantitativa. Se aplicó el instrumento diseñado a una muestra no probabilística intencional de 1.000 estudiantes de tercer año básico del sector municipal de Puerto Montt, representando 59,3\% de la matrícula total inicial del año 2013 para los terceros básicos de esa comuna. El 50,2\% de las estudiantes es de sexo femenino y el $49,8 \%$ de sexo masculino, manteniéndose una proporción similar para evitar sesgo de género. El 90,3\% cursa el tercer año básico con ocho o nueve años de edad que corresponde a la edad apropiada para el curso, siendo el 9,7\% restante estudiantes que cursan con una sobreedad de hasta cinco años. El $84 \%$ de los estudiantes no presenta ninguna necesidad educativa especial diagnosticada, mientras que $16 \%$ se encuentra con alguno de los siguientes diagnósticos: trastorno específico del aprendizaje, déficit atencional, trastorno específico del lenguaje, rango intelectual entre 60 y 79 puntos. Se excluyó de la muestra a todo estudiante que presentara diagnósticos que impidieran contestar el instrumento tal como se encuentra diseñado (discapacidad visual, auditiva, parálisis cerebral, autismo o discapacidad intelectual moderada).

En la investigación participaron 34 establecimientos municipales de Puerto Montt que representan $56 \%$ del total comunal. Los establecimientos se sectorizaron incluyendo 13 establecimientos rurales y 21 urbanos.

La aplicación fue realizada por psicopedagogas de los establecimientos previa capacitación en tres jornadas de no más de 15 personas cada una. Las capacitaciones se llevaron a cabo durante noviembre de 2013. De esta forma se cauteló que al momento de ser evaluados los estudiantes tuvieran la cobertura curricular correspondiente al nivel cursado.

El instrumento original diseñado corresponde a una rúbrica analítica de desempeño de cuatro niveles y 25 ítems. Se estableció un número par para evitar la tendencia del evaluador a puntuar un nivel intermedio. El instrumento consta de un manual en donde el evaluador conoce la descripción de la habilidad que está evaluando y la descripción por cada nivel de desempeño, un protocolo por estudiante en el que se registra el nivel de desempeño y observaciones, y un set de textos y material original que se aplica para obtener las respuestas de los estudiantes. Previo a la evaluación se solicitó consentimiento por escrito a cada apoderado para la aplicación a los estudiantes.

Los datos fueron procesados en el software S.P.S.S. versión 11.0 para Windows XP. Se estableció la propiedad psicométrica de confiabilidad por medio de la prueba de Alpha 
de Cronbach (Gliem \& Gliem, 2003; Hernández, Fernández y Baptista, 2006; Morales, 2013), la propiedad psicométrica de validez de contenido mediante un análisis factorial exploratorio y la validez de criterio y poder de discriminación por medio de la correlación entre los puntajes del Instrumento y del test de autoestima escolar (Marchant, Haeussler y Torretti, 2002), el promedio de lenguaje y la discriminación entre estudiantes con y sin necesidades educativas especiales.

\section{ANÁLISIS DE RESULTADOS}

\subsection{DISEÑO DEL INSTRUMENTO}

El conjunto de redes semánticas obtenido mediante el Análisis de Redes Semánticas utilizado para determinar las habilidades reconocidas por la comunidad del sector municipal de Puerto Montt, demostró un valor J igual a 80 conceptos vinculados a habilidades de comprensión lectora. El valor M de cada uno de estos conceptos determinó las siguientes habilidades como las más representativas del concepto: Vocabulario (94), Inferir (46), Fluidez (42), Velocidad (36), Identificar Ideas Principales (36), Extraer Información Relevante (33), Análisis (30), Decodificación (28), Síntesis (28) y Secuenciación (28).

El juicio de expertos determinó $78 \%$ de acuerdo entre los cuatro jueces respecto de los ítems diseñados y las habilidades que pretenden medir. Los ítems, 1, 2, 7, 8, 10, 12, 13, 15, 16 y 18 lograron $100 \%$ de acuerdo. Los ítems 4 y 11 lograron $25 \%$. Respecto de la batería de textos, cada juez asignó un valor entre 1 y 3 , donde 1 implica deficiencias que hacen imposible su aplicación, 2 aplicación pero con modificaciones y 3 aplicable. Los jueces evaluaron Ortografía de los textos, Redacción, Tipografía y Sesgos. Todos los criterios obtuvieron puntajes cercanos a 3,00 excepto Redacción, que obtuvo 2,50. Se consideraron todas las sugerencias de enfoque y redacción para la elaboración del instrumento final.

\subsection{PROPIEDADES PSICOMÉTRICAS DEL INSTRUMENTO}

La confiabilidad se estimó por medio de la prueba estadística Alpha de Cronbach obteniéndose un puntaje de .773 para 25 ítems, lo que se considera aceptable (George \& Mallery, 2003, Hernández et al., 2006; Morales, 2013).

Si bien ninguno de los ítems presenta correlaciones bajas o negativas, los ítems 7 y 8 presentan las más bajas entre el total. Ambos corresponden a la Ficha de Actitud Lectora que evalúa con cuatro ítems la motivación del estudiante por leer, la frecuencia de la lectura y el uso de la biblioteca. Se decide eliminar los ítems, 7, 8, 9 y 10 tomando en consideración a) el aporte que realizan a la confiabilidad del instrumento, b) los fundamentos teóricos que atribuyen el desarrollo de las habilidades medido a partir de aspectos contextuales (biblioteca, motivación) y c) la contribución a la aplicación del instrumento y la fatiga.

De esta forma el instrumento quedó constituido por 21 ítems. El nuevo análisis de Alpha de Cronbach estima un puntaje de .778, manteniéndose la confiabilidad aceptable.

Respecto de la dificultad del instrumento, este presenta un puntaje mínimo posible de 25 (muy bajo nivel de comprensión lectora) y un máximo posible de 100 (alto nivel de comprensión lectora). La media aritmética es 75 (nivel intermedio de comprensión 
lectora) y la dificultad media obtenida por los estudiantes fue de 60 puntos con una desviación típica de 9,49. El instrumento, por tanto, resulta exigente para el nivel pero a la vez permite que exista un grupo de estudiantes que logra puntajes altos, como se observa en el histograma (ver Figura 1), el que se asemeja a la campana teórica de Gauss.

Figura 1. Histograma test total con 21 elementos

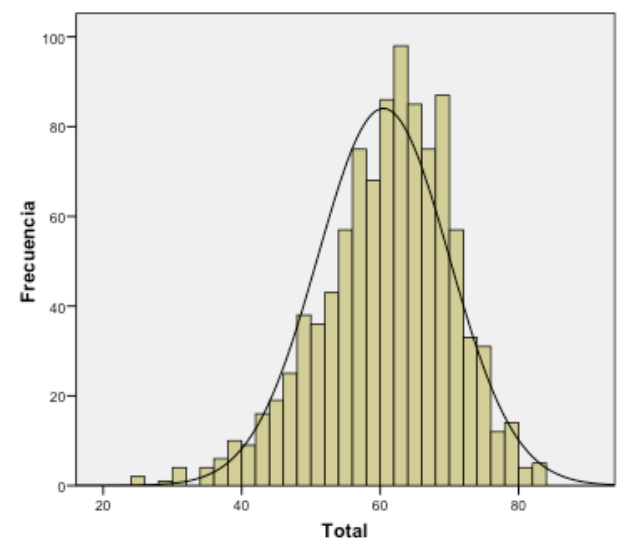

Fuente: exportado desde el programa S.P.S.S. para Windows XP versión 11.0.

Respecto de la consistencia interna se observan bajas correlaciones. Morales (2013) establece que en la matriz no debiera haber correlaciones bajo .300 y que, de existir, se puede atribuir a la homogeneidad de la muestra. En este sentido y debido al carácter exploratorio de la investigación se establece aceptable una correlación de .250. Además, se considera que, al ser todos los establecimientos del sector municipal, el nivel socioeconómico de la muestra es relativamente homogéneo.

Con los antecedentes previamente planteados se determina que el instrumento cuenta con características de confiabilidad aceptables para el sector municipal de Puerto Montt.

Para determinar la discriminación del instrumento se estima la asociación de respuesta entre los estudiantes que presentan necesidades educativas especiales y aquellos que no presentan. La hipótesis es que el instrumento debe ser capaz de discriminar entre ambos grupos, para ello se aplica la prueba Chi cuadrado de independencia. Para estos efectos se recodificó la variable puntaje total del instrumento en una variable ordinal donde los estudiantes con puntaje bajo 60 se ubicaron en la categoría bajo nivel de comprensión lectora y aquellos con puntaje igual superior a 60 en la categoría de alto nivel de comprensión lectora.

La prueba establece un nivel de significancia .000 , por tanto al ser $\mathrm{p}<.005$ se rechaza la hipótesis nula y se asume que las variables son dependientes, existiendo por tanto una relación entre las Necesidades Educativas Especiales de los estudiantes y su nivel de comprensión lectora (ver Tabla 1).

Se concluye, por tanto, que el instrumento logra discriminar entre aquellos estudiantes con necesidades educativas especiales y aquellos que no, tomando en cuenta su nivel de comprensión lectora. 
Tabla 1. Prueba Chi-cuadrado para NEE y nivel de Comprensión Lectora

\begin{tabular}{|l|c|c|c|c|c|}
\cline { 2 - 6 } \multicolumn{1}{c|}{} & Valor & Gl & $\begin{array}{c}\text { Sig. asintótica } \\
\text { (bilateral) }\end{array}$ & $\begin{array}{c}\text { Sig. exacta } \\
\text { (bilateral) }\end{array}$ & $\begin{array}{c}\text { Sig. exacta } \\
\text { (unilateral) }\end{array}$ \\
\hline Chi-cuadrado de Pearson & $93,831(\mathrm{~b})$ & 1 &, 000 & & \\
\hline Corrección por continuidad(a) & 91,376 & 1 &, 000 & & \\
\hline Razón de verosimilitudes & 73,941 & 1 &, 000 & &, 000 \\
\hline Estadístico exacto de Fisher & & & & &, 000 \\
\hline Asociación lineal por lineal & 93,738 & 1 &, 000 & & \\
\hline $\mathrm{N}^{\circ}$ de casos válidos & 1000 & & & & \\
\hline
\end{tabular}

a Calculado solo para una tabla de $2 \times 2$.

b 0 casillas $(, 0 \%)$ tienen una frecuencia esperada inferior a 5. La frecuencia mínima esperada es 21,04.

Fuente: exportado desde el Programa S.P.S.S. para Windows XP versión 11.0.

La validez de criterio se estimó comparando el Rendimiento en el Test con el Promedio de notas de Lenguaje y Comunicación, y el Rendimiento en el Test con el resultado del test de autoestima escolar (TAE) de Marchant et al. (2002) validado para población chilena.

Se aplicó la Prueba T Student para muestras independientes. Inicialmente la Prueba de Levene muestra un nivel de significación $p>.005$, por tanto se asume que las varianzas de ambas muestras son iguales. El nivel de significancia bilateral de la Prueba $\mathrm{T}$ de Student es menor a .005 , por tanto se rechaza la hipótesis nula y se asume que existen diferencias significativas en el rendimiento de los estudiantes en Lenguaje y Comunicación según la variable resultado del test (ver Tabla 2).

El mismo procedimiento se aplicó para determinar la independencia de las variables Test de Autoestima Escolar y Resultado del Instrumento. La relación entre autoestima escolar y comprensión lectora se encuentra estudiada (Valles, 2005; Ripoll, Aguado y Díaz, 2007; Marchant et al., 2007), por tanto se esperaría que existiera una relación entre ambos puntajes.

La Prueba de Levene para igualdad de varianzas demuestra un valor superior a .005 , por tanto se asume que las varianzas son iguales y se trabaja con la primera fila de resultados. De esta forma, la Prueba $\mathrm{T}$ para igualdad de medias determina un valor inferior a .005 , por tanto se asume que existen diferencias significativas en el resultado del test de autoestima escolar según el resultado del test de comprensión lectora (ver Tabla 3). En consecuencia, se concluye que existe una correlación significativa entre el resultado del instrumento de comprensión lectora y el rendimiento en lenguaje y la autoestima escolar. 
Tabla 2. Prueba T para comparar medias de la variable Rendimiento y Resultado del Test

\begin{tabular}{|c|c|c|c|c|c|c|c|c|c|c|}
\hline & \multicolumn{2}{|c|}{$\begin{array}{c}\text { Prueba de } \\
\text { Levene para } \\
\text { la igualdad de } \\
\text { varianzas }\end{array}$} & \multicolumn{7}{|c|}{ Prueba $\mathrm{T}$ para la igualdad de medias } \\
\hline & & \multirow{2}{*}{$\begin{array}{c}F \\
\text { Inferior }\end{array}$} & \multirow{2}{*}{\begin{tabular}{|c|} 
Sig. \\
Superior
\end{tabular}} & \multirow{2}{*}{\begin{tabular}{|c|}
$\mathrm{T}$ \\
Inferior
\end{tabular}} & \multirow{2}{*}{\begin{tabular}{|c|} 
Gl \\
Superior \\
\end{tabular}} & \multirow{2}{*}{$\begin{array}{c}\begin{array}{c}\text { Sig. } \\
\text { (bilateral) }\end{array} \\
\text { Inferior }\end{array}$} & \multirow{2}{*}{$\begin{array}{l}\text { Diferencia } \\
\text { de medias }\end{array}$} & \multirow{2}{*}{$\begin{array}{c}\begin{array}{c}\text { Error } \\
\text { típ. de la } \\
\text { diferencia }\end{array} \\
\text { Inferior }\end{array}$} & \multicolumn{2}{|c|}{$\begin{array}{l}95 \% \text { Intervalo de } \\
\text { confianza para la } \\
\text { diferencia }\end{array}$} \\
\hline & & & & & & & & & Superior & Inferior \\
\hline \multirow[t]{2}{*}{ Promedio } & $\begin{array}{l}\text { Se han } \\
\text { asumido } \\
\text { varianzas } \\
\text { iguales }\end{array}$ & 1,780 & ,182 & $-7,405$ & 978 & ,000 & $-4,884$ & ,660 & $-6,179$ & $-3,590$ \\
\hline & $\begin{array}{l}\text { No se han } \\
\text { asumido } \\
\text { varianzas } \\
\text { iguales }\end{array}$ & & & $-7,889$ & 184,942 & ,000 & $-4,884$ & 619 & $-6,106$ & $-3,663$ \\
\hline
\end{tabular}

Fuente: exportado desde el Programa S.P.S.S. para Windows XP Versión 11.0.

Tabla 3. Prueba T para comparar medias de la variable

Test de Autoestima Escolar y Resultado del Test

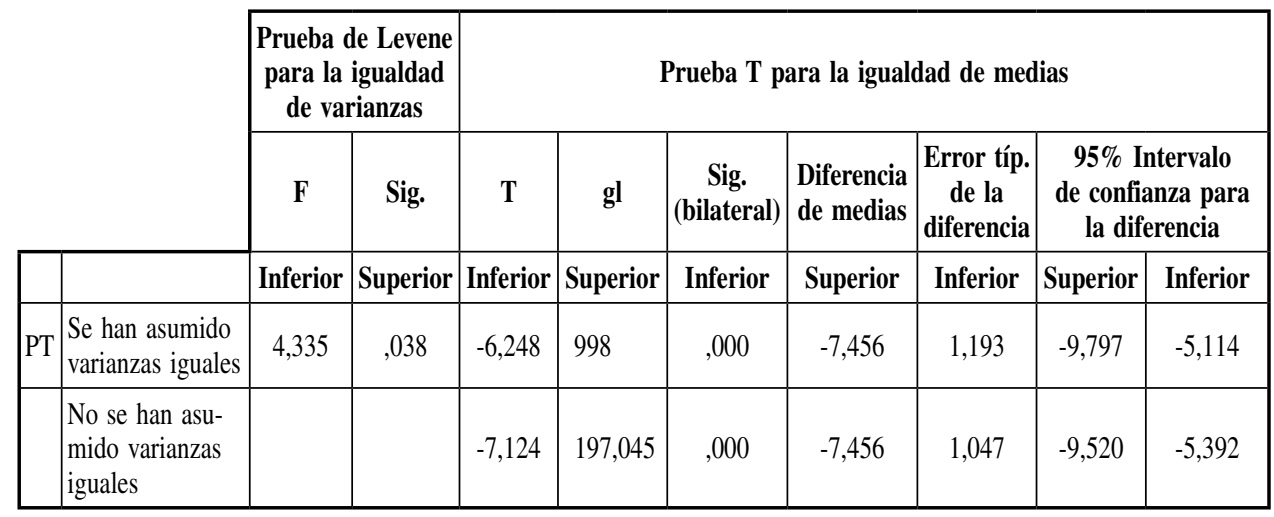

Fuente: exportado desde el Programa S.P.S.S. para Windows XP Versión 11.0.

Para la validez de constructo se realizó un análisis factorial exploratorio, con la finalidad de observar cómo se agrupan los ítems del test y si esta agrupación guarda relación con los elementos teóricos revisados. Para esto se utilizó la extracción de componentes principales.

La medida de adecuación muestral de Kaiser-Meyer-Olkin demuestra un buen ajuste, por tanto puede procederse al análisis factorial. Asimismo, la Prueba de Esfericidad de Barlett muestra una significancia inferior a .005 , de esta forma se asume que la matriz de correlación es significativamente distinta de la matriz de identidad. 
Respecto de las comunalidades y debido al carácter exploratorio del estudio, se establece como adecuados, aquellos ítems que sean superiores a .500 (Hair, Anderson y Tatham, 1999). Se observan buenos pesos para los ítems 1, 2, 4, 15, 17, 18, 20 y 23. Se mantienen todos los ítems, ya que se hipotetiza la baja comunalidad de los demás por la homogeneidad de la muestra.

Al realizar un primer análisis factorial se observa una estructura monofactorial, por ello se procede a realizar una rotación Varimax, con esto se observa una estructura de seis factores con distribución de los ítems cuyo análisis se presenta en la Tabla 4.

La varianza total explicada de los seis factores es del 47,6\%, a base de esto se hipotetiza que existe una varianza debida a errores de medición correspondiente a 52,4\%. Esto podría explicarse por la extensión del instrumento y por la homogeneidad de la muestra (Morales, 2013).

Tabla 4. Agrupación de los ítems según factores y sus comunalidades

\begin{tabular}{|c|c|c|c|c|c|c|c|}
\hline \multirow{2}{*}{ Ítems } & \multicolumn{6}{|c|}{ Factores } & \multirow{2}{*}{ Comunalidades } \\
\hline & 1 & 2 & 3 & 4 & 5 & 6 & \\
\hline 8. Relacionar conocimientos previos & ,346 & ,236 &,- 060 & ,054 & ,381 &,- 081 & 334 \\
\hline 9. Extraer información implícita & ,472 & ,207 &, 100 &,- 052 &, 165 &,- 177 & ,337 \\
\hline 11. Extraer información explícita & ,731 & ,074 & ,074 &, 117 & ,049 & ,103 &, 572 \\
\hline 12. Extraer información implícita & 667 &,- 015 &, 136 &, 114 & ,007 &,- 070 & ,482 \\
\hline 13. Interpretar lenguaje figurado & ,660 & ,133 &, 143 &, 157 &,- 016 &, 117 & ,512 \\
\hline 21. Formulación de preguntas & ,362 & ,289 & 002 & ,138 & 092 & 323 & ,347 \\
\hline 1. Decodificación & ,158 & 843 &, 120 & 087 & 022 &,- 018 &, 758 \\
\hline 2. Entonación & , 150 & 854 & , 130 & ,077 & 060 &,- 034 & ,779 \\
\hline 14. Subrayar &,- 039 & ,110 & ,696 &,- 114 & 059 & ,113 & ,528 \\
\hline 15. Descripción de contextos & ,171 & ,023 & ,584 & ,095 & ,156 &,- 069 & 409 \\
\hline 16. Visualizar & , 163 & ,112 & 655 & ,193 &,- 046 &,- 029 & ,508 \\
\hline 17. Descripción de personajes & , 197 & 016 & 429 & 312 & 219 &, 159 & 394 \\
\hline 3. Expresión de opiniones & , 166 & 119 &, 163 &, 584 & 093 &, 195 & 455 \\
\hline $\begin{array}{l}\text { 4. Determinar significados } \\
\text { desconocidos, contexto }\end{array}$ &, 140 &,- 105 &,- 020 &, 710 & ,016 &,- 148 &, 557 \\
\hline 5. Releer &, 138 &, 254 & ,218 &, 458 &, 013 &,- 385 &, 489 \\
\hline $\begin{array}{l}\text { 18. Determinar significados } \\
\text { desconocidos palabra }\end{array}$ & ,000 & ,209 & ,052 &, 524 & ,255 & ,118 & ,400 \\
\hline 6. Localizar información & ,205 & ,218 &, 029 &, 104 & ,232 &,- 466 &, 372 \\
\hline $\begin{array}{l}\text { 7. Determinar significados } \\
\text { desconocidos, prefijos }\end{array}$ &, 019 &,- 045 &, 116 & ,207 &, 585 & ,017 & ,401 \\
\hline 19. Interpretación de metáforas &,- 022 &, 182 &, 020 &, 102 & 665 & 240 &, 545 \\
\hline 20. Uso de imágenes &, 125 &,- 078 &, 172 &,- 059 &, 571 &,- 283 & ,461 \\
\hline 10. Reconstrucción de secuencias & 211 & 072 &, 182 & 113 & 143 &, 505 &, 370 \\
\hline
\end{tabular}

Fuente: elaborado en base a las tablas exportadas desde el Programa S.P.S.S. para Windows XP versión 11.0. 
El factor 1 queda conformado por los ítems Relacionar conocimientos previos $\left(\mathrm{N}^{\mathrm{o}} 8\right)$, Extraer información explícita ( $\mathrm{N}^{\mathrm{o}}$ 9), Extraer información implícita ( $\mathrm{N}^{\mathrm{o}}$ 11), Extraer información explícita ( $\left.\mathrm{N}^{\mathrm{o}} 12\right)$, Formulación de Preguntas $\left(\mathrm{N}^{\mathrm{o}} 21\right)$, explicando, en su conjunto, $19,6 \%$ de la varianza.

Debido al carácter de obtención de información que tienen los ítems, se denomina al factor "Extracción de Información" quedando definido así como compuesto por ítems que permiten al lector extraer información del texto. El Currículum chileno (MINEDUC, 2013) agrupa también en los objetivos 3 y 5 las habilidades de extracción de información y la relación de conocimientos previos, siendo este último ítem ubicado dentro de aquellos que permiten al alumno obtener más información para la comprensión. Asimismo, León y Pérez (2003) establecen una categoría de habilidades de lectura que el lector pone en práctica por la necesidad de saber. Dentro de estas se puede ubicar la formulación de una pregunta y la interpretación del lenguaje figurado.

El segundo factor queda definido por los ítems Decodificación $\left(\mathrm{N}^{\mathrm{o}} 1\right)$ y Entonación $\left(\mathrm{N}^{\mathrm{o}} 2\right)$, denominándose como Fluidez Lectora, de acuerdo con los postulados de Jiménez y O'Shanaham (2008), Marchant et al. (2012) y Nathan \& Stavonich (1991). explicando el $6,6 \%$ de la varianza.

El tercer factor queda definido por los ítems 14. Subrayar, 15. Descripción de contextos, 16. Visualizar y 17. Descripción de Personajes, explicando el 5,9\% de la varianza. A esta agrupación se le denominó "Estrategias Durante la Lectura", de acuerdo con los postulados de Gutiérrez-Braojos y Salmerón (2012) El Subrayar y el visualizar se identifican como estrategias de representación visual, mientras que la Descripción necesariamente requiere de la representación mental de lo que se está leyendo.

El cuarto factor queda definido por los ítems Expresión de Opiniones ( $\mathrm{N}^{\mathrm{o}} 3$ ), Determinar significados desconocidos utilizando el contexto $\left(\mathrm{N}^{\circ} 4\right)$, Releer $\left(\mathrm{N}^{\circ} 5\right)$ y Deducir significados a partir de la estructura de la palabra $\left(\mathrm{N}^{\mathrm{o}} 18\right)$. A este factor, que explica el 5,5\% de la varianza, se le denomina "Estrategias después de la lectura", siguiendo también los postulados de Gutiérrez-Braojos y Salmerón (2012), Fonseca et al. (2014) y Menti y Rosemberg (2014). Dichas estrategias se ponen en práctica una vez que se ha finalizado la lectura para compartir lo que se ha leído (Expresión de opiniones) o reconocer si se ha obviado información (releer, determinar significados utilizando el contexto y estructura de la palabra).

El quinto factor queda compuesto por los ítems Deducir significados utilizando prefijos ( $\left.\mathrm{N}^{\mathrm{o}} 11\right)$, Interpretación de Lenguaje Figurado ( $\left.\mathrm{N}^{\mathrm{o}} 23\right)$, Uso de Imágenes $\left(\mathrm{N}^{\mathrm{o}} 24\right)$ y Localizar información ( $\mathrm{N}^{\mathrm{o}} 5$ ), explicando el 4,9\% de la varianza. A este factor se le denominó Interpretación de Textos No Literarios, basándose en la importancia que cobra este tipo de textos y su abordaje en el proceso de la lectura (Pérez, 2005; Méndez, 2006; Arancibia y Henríquez, 2008; Castillo, 2011) En ese sentido se propone cambiar el nombre al ítem Interpretación del Lenguaje Figurado, por Interpretación de Metáforas, para darle un contexto más apropiado a un texto no literario y para diferenciarlo del lenguaje figurado evaluado en el ítem 17.

El sexto factor explica el 4,8\% de la varianza y se encuentra compuesto por el ítem 14. A pesar de esto, se decide mantener el ítem y por tanto el factor, bajo el nombre de Secuenciación, porque en el trabajo de Redes Semánticas fue identificado por los profesores como una de las habilidades importantes a la hora de la comprensión lectora. Asimismo, se encuentra presente en el Currículum chileno (MINEDUC, 2013). 
Los seis factores identificados permiten abordar todos los ítems, y de igual forma explican el constructo de comprensión lectora revisado a partir del Currículum vigente, la bibliografía revisada y las necesidades de los docentes y profesionales del sistema.

\section{CONCLUSIONES}

El instrumento presentado y sometido a análisis fue construido tomando como base el Currículum chileno y habilidades de comprensión lectora definidas por un conjunto de docentes y autoridades vinculadas a la educación de niños y niñas del sector municipal de Puerto Montt. Se definieron las habilidades de Inferencia, Decodificación, Vocabulario y Secuenciación que posteriormente fueron complementadas y contrastadas con una revisión bibliográfica tomando como base postulados de León y Pérez (2003), Gutiérrez-Braojos y Salmerón (2012), el Ministerio de Educación chileno (2013) y Fonseca et al. (2014) Así, se llega a identificar 25 habilidades de comprensión lectora.

El Test de Habilidades de Comprensión Lectora presenta un nivel de confiabilidad aceptable para 21 ítems medido con un Alpha de Cronbach .778, un puntaje mínimo posible de 21, un puntaje máximo de 100 y una media de respuesta de 60 . Se excluyeron 4 ítems, que tendían a disminuir el coeficiente de confiabilidad y no contribuían al desarrollo del instrumento.

Respecto de las propiedades psicométricas, se presentan niveles adecuados de validación, comenzando por una validación de contenidos a partir de Juicio de Expertos utilizando el Método de Agregados Individuales, resultando la mayoría de los ítems como aceptables. Solo dos ítems lograron niveles no aceptables, los que fueron revisados, reformulados y sometidos a prueba.

El instrumento cuenta con validez de criterio, estableciendo correlaciones significativas entre el resultado del test y los siguientes criterios: test de autoestima escolar y rendimiento en la asignatura de lenguaje. Asimismo, el instrumento logra diferenciar entre estudiantes con y sin necesidades educativas especiales.

Finalmente, se realiza un análisis factorial exploratorio que identifica seis factores que logran asociarse a los postulados teóricos que establece la teoría sobre la base de cómo fue diseñado el instrumento. Los factores identificados son Extracción de Información, Habilidades Fonológicas, Estrategias Durante la Lectura, Estrategias Después de la Lectura, Interpretación de Textos No Literarios y Secuenciación.

El instrumento cuenta con criterios psicométricos de confiabilidad y validez que permiten afirmar que el conjunto de ítems contribuye a determinar un nivel de comprensión lectora e identificar a estudiantes que podrían requerir de apoyos en el mencionado proceso.

De las limitaciones del estudio es importante señalar que los niveles aceptados para las correlaciones siguen siendo bajos para los supuestos teóricos establecidos y mencionados en la discusión. Para controlar esto se requiere revisar lo siguiente a) Precisar las instrucciones del manual de aplicación para mejorar la forma de entregar las instrucciones por parte de los evaluadores, y b) Ampliar la muestra a niveles socioeconómicos altos, pues en esta investigación la aplicación del instrumental se concentró en su mayoría en niveles socioeconómicos bajos. Existe evidencia que señala que las diferencias económicas influyen en el nivel de comprensión (Fonseca et al., 2014). 
Se puede proyectar una nueva validación del instrumento, considerando los dos elementos anteriormente señalados. Asimismo, el instrumento con los criterios de confiabilidad y validez de criterio permitiría ampliar estudios para ser usado como instrumento de evaluación previo o posterior a metodologías de estudio. Finalmente se puede someter a validación de criterio junto con otros instrumentos de comprensión lectora como son las pruebas psicopedagógicas Evalúa-3 (García, González y García, 2004) .

\section{REFERENCIAS BIBLIOGRÁFICAS}

Ahmadi, M. (2012). Impacts of learning reading strategy on student's reading comprehension proficiency. The International Journal of Language and Applied Linguistics World IJLLALW, vol. 1 (1), 72-87.

Allende, F., Condemarín, M. y Milicic, N. (2012). Prueba CLP formas paralelas. Manual para la aplicación de la prueba de comprensión lectora de complejidad lingüística progresiva: 8 niveles de lectura ( $9^{\text {na }}$ Ed.). Santiago: Ediciones UC.

Arancibia, B. y Henríquez, M. (2008). La lectura de textos no literarios en los textos escolares de lenguaje y comunicación de segundo ciclo básico del sistema escolar chileno: La apreciación de los profesores. Enunciación, vol. 13 (1), 61-70.

Barreyro, J. y Molinari, C. (2005). Diferencias individuales en la comprensión de textos: Inferencias y capacidad de la memoria de trabajo. Anuario de Investigaciones, vol. 13 (1), 43-50.

Bizama, M., Arancibia, B. y Sáez, K. (2013). Intervención psicopedagógica temprana en conciencia fonológica como proceso metalingüístico a la base de la lectura en niños de 5 a 6 años socialmente vulnerables. Estudios Pedagógicos, vol. 39 (2), 25-39.

Canet-Juric, L., Burin, D., Andrés, M. y Urquijo, S. (2013). Perfil cognitivo de niños con rendimientos bajos en comprensión lectora. Anales de Psicología, vol. 29 (3), 996-1005.

Cassany, D., Luna, M. y Sanz, G. (2000). Enseñar lengua (5 ${ }^{\text {ta }}$ Ed.). México D.F.: Graò.

Castillo, J. (2011). Mediación de la comprensión lectora de textos literarios en educación básica. Paradígma, vol. 32 (1), 103-122.

CERLALC-UNESCO (2012). El libro en cifras: Boletín estadístico del libro en Iberoamérica. Bogotá: Edición Juliana Camacho. Recuperado el 25 de julio de 2013 desde http://cerlalc.org/wpcontent/uploads/2013/03/LEC_I_Def.pdf

Corral, Y. (2009). Validez y confiabilidad de los instrumentos de investigación para la recolección de datos. Revista Ciencias de la Educación, vol. 19 (33), 228-247.

Escudero, I. (2010). Las inferencias en la comprensión lectora: Una ventana hacia los procesos cognitivos en segundas lenguas. Revista Nebrija de Lingüística Aplicada a la Enseñanza de las Lenguas, vol. 7 (1), 1-20.

Fonseca, L., Pujals, M., Lasala, E., Lagomarsino, I., Migliardo, G., Aldrey, A., Buonsanti, L. y Barreyro, J. (2014). Desarrollo de habilidades de comprensión lectora en niños de escuelas de distintos sectores socioeconómicos. Neuropsicología Latinoamericana, vol. 6 (1), 41-50.

García, E. (1993). La comprensión de textos. Modelo de procesamiento y estrategias de mejora. Didáctica, vol. 5 (1), 87-113.

García, J., González, D. y García, B. (2004). Batería Psicopedagógica Evalúa-3. Manual Versión 2.0 (Ed. adaptada para Chile). Santiago: EOS.

George, D. \& Mallery, P. (2003). SPSS for WINDOWS step by step. A simple guide and reference. 11.0 Update (4 ${ }^{\text {th }}$ Ed.). Boston: Allyn \& Bacon.

Gliem, J. \& Gliem, R. (2003). Calculating, interpreting and reporting Cronbach's alpha reliability coefficient for likert-type scales. 2003 Midwest Research to Practice Conference in Adult, Continuing, and Community Education, 82-88. Retrieved August 24, 2013 from https://scholarworks. iupui.edu/bitstream/handle/1805/344/Gliem+\&+Gliem.pdf?sequence $=1$ 
Goikoetxea, E. (2012). Las dificultades específicas de aprendizaje en el albor del siglo XXI. Relieve, vol. 18 (1), 1-18.

Gómez, E., Defior, S. y Serrano, F. (2011). Mejorar la fluidez lectora en dislexia: Diseño de un programa de intervención en español. Escritos de Psicología, vol. 4 (2), 65-73.

Gutierrez-Braojos, C. y Salmerón, H. (2012). Estrategias de comprensión lectora: Enseñanza y evaluación en educación primaria. Profesorado, vol. 16 (1), 183-202.

Hair, J., Anderson, R. y Tatham, W. (1999). Análisis multivariante (5 ${ }^{\text {ta }}$ Ed.) Madrid: Pentice.

Hernández, R., Fernández, C. y Baptista, P. (2006). Metodología de la investigación (4 ${ }^{\text {ta }}$ Ed.). México D.F.: Mc Graw Hill.

Hinojosa, G. (2008). El tratamiento estadístico de las redes semánticas naturales. Revista Internacional de Ciencias Sociales y Humanidades SOCIOTAM, vol. 18 (1), 133-154.

IEA (2012). PIRLS - TIMSS 2011. Estudio internacional de progreso en comprensión lectora, matemáticas y ciencias (Vol. I). Madrid: Ministerio de Educación, Cultura y Deporte. Recuperado el 20 de mayo de 2013 desde http://www.mecd.gob.es/dctm/inee/internacional/pirlstimss2011vol 1. pdf?documentId=0901e72b8146f0ca

Jiménez, J. y O'Shanahan, I. (2008). Enseñanza de la lectura: De la teoría y la investigación a la práctica educativa. Revista Iberoamericana de Educación, vol. 45 (5), 1-22.

León, J. y Pérez, O. (2003). Taxonomías y tipos de inferencia. En J. León (Coord.), Conocimiento y discurso. Claves para inferir y comprender (pp. 45-66). Madrid: Pirámide.

Lyon, G., Shaywitz, S. \& Shaywitz, B. (2003). A definition of dyslexia. Annals of Dyslexia, vol. 53 (1), 1-14.

Marchant, T., Recart, I., Cuadrado, B. y Sanhueza, J. (2012). Pruebas de dominio lector para alumnos de enseñanza básica (4 ${ }^{\text {ta }}$ Ed.). Santiago: Ediciones UC.

. Lucchini, G. y Cuadrado, B. (2007). ¿Por qué leer bien es importante?: Asociación del dominio lector con otros aprendizajes. Psykhe, vol. 16 (2), 3-16.

Ediciones UC.

Haeussler, I. y Torretti, A. (2002). TAE: Batería de test de autoestima escolar. Santiago:

Méndez, S. (2006). Comprensión lectora y textos literarios: Una propuesta psicopedagógica. Educación, vol. 30 (1), 141-155.

Menti, A. y Rosemberg, C. (2014). ¿Cómo se llama? ¿Qué significa? Análisis de las interacciones docente-alumno en el tratamiento de palabras desconocidas. Psykhe, vol. 23 (1), 1-13.

MINEDUC (2013). Lenguaje y comunicación. Programa de estudio para tercer año básico. Santiago: Gobierno de Chile.

. (2012). Síntesis de resultados SIMCE 2011. Santiago: Gobierno de Chile. Recuperado el 20 de mayo de 2013 desde http://www.agenciaeducacion.cl/wp-content/files_mf/folleto_sintesis_web_2012.pdf

. (2009). Resumen de resultados PISA 2009 Chile. Santiago: Gobierno de Chile. Recuperado el 20 de mayo de 2013 desde http://www.agenciaeducacion.cl/wp-content/files_mf/ resumenderesultadospisa2009chile.pdf

Mora, J. y Aguilera, A. (Coords.) (2000). Atención a la diversidad en educación: Dificultades en el aprendizaje del lenguaje, de las matemáticas y en la socialización. Sevilla: Kronos.

Morales, P. (2013). El análisis factorial en la construcción e interpretación de tests, escalas y cuestionarios. Madrid: Universidad Pontificia Comillas. Recuperado el 20 de mayo de 2013 desde http://www.upcomillas.es/personal/peter/investigacion/AnalisisFactorial.pdf

Muñoz, C. y Schelstraete, M. (2008). Decodificación y comprensión de lectura en la edad adulta: ¿Una relación que persiste? Iberoamericana de Educación, vol. 45 (5), 1-8.

Nathan, R. \& Stavonich, K. (1991). The causes and consequences of differences in reading fluency. Theory into Practice, vol. 30 (3), 176-184.

OECD (2013). PISA 2012 Assessment and analytical framework: Mathematics, reading, science, problem solving and financial literacy. Retrieved on August 28, 2013 from http://dx.doi. org/10.1787/9789264190511- 
(2003). Marcos teóricos de PISA 2003. Conocimientos y destrezas en matemáticas, lectura, ciencias y solución de problemas. Madrid: Ministerio de Educación y Ciencia.

Paredes, J. (2006). Decodificación y lectura. Actualidades Investigativas en Educación, vol. 6 (2), 1-22.

Pascual, G., Goikoetxea, E., Corral, S., Ferrero, M. y Pereda, V. (2014). La enseñanza recíproca en las aulas: Efectos sobre la comprensión lectora en estudiantes de primaria. Psykhe, vol. 23 (1), 1-12.

Pérez, M. (2005). Evaluación de la comprensión lectora: Dificultades y limitaciones. Revista de Educación, (Número Extraordinario), 121-138. Recuperado el 22 de febrero de 2014 desde http:// www.oei.es/evaluacioneducativa/evaluacion_comprension_lectora_perez_zorrilla.pdf

Rathvon, N. (2004). Early reading assessment: A practitioner's handbook. New York: The Guilford Press.

Ripoll, J. y Aguado, G. (2014). La mejora de la comprensión lectora en español. Un meta-análisis. Revista de Psicodidáctica, vol. 19 (1), 27-44.

y Díaz, M. (2007). Mejora de la comprensión lectora mediante el entrenamiento en la construcción de inferencias. Puls, vol. 30 (1), 233-245.

Rosas, R., Medina, L., Meneses, A., Cuchacovich, S. y Escobar, P. (2011). Construcción y validación de una prueba de evaluación de competencia lectora inicial basada en computador. Pensamiento Educativo, vol. 48 (1), 43-61.

Sánchez, S. (2008). La construcción social de dificultades de aprendizaje en las prácticas educativas. Revista Brasileira de Educação Especial, vol. 14 (3), 347-364.

Soriano-Ferrer, M., Sánchez-López, P., Soriano-Ayala, E. y Nievas-Cazorla, F. (2013). Instrucción en estrategias de comprensión lectora mediante enseñanza recíproca: Efectos del agrupamiento de los estudiantes. Anales de Psicología, vol. 29 (3), 848-854.

Silvia, C. e Aparecida, S. (2011). Desempenho cognitivo-linguístico de escolares com distúrbio de apredizagem. Psicología em Estudo, vol. 16 (1), 131-137.

UNESCO (2008). SERCE. Segundo estudio regional comparativo y explicativo. Los aprendizajes de estudiantes de América Latina y el Caribe. Santiago: Salesianos Impresiones.

Valles, A. (2005). Comprensión lectora y procesos psicológicos. Liberabit, vol. 11 (1), 49-61.

Vera-Noriega, J. (2005). Redes semánticas: Aspectos teóricos, técnicos, metodológicos y analíticos. Ra Ximhai, vol. 1 (3), 439-451. 
